\title{
Acquired Hemophilia A Simulating Retropharyngeal Abscess: Importance of Differential Diagnosis of Neck Masses before Surgery
}

\author{
Isabel López-Sánchez¹, José-Ramón Alba-García1, Cristina Vázquez-Romero1, \\ Miguel Armengot-Carceller ${ }^{2 *}$ \\ ${ }^{1}$ ENT Department, General and University Hospital, Valencia, Spain \\ ${ }^{2}$ Rhinology Unit, ENT Department, General and University Hospital.Valencia University, Valencia, Spain \\ Email: isa.lopez.85@gmail.com, joseramon43@hotmail.com, crisvazquez50@hotmail.com, \\ miguel.armengot@uv.es
}

Received 14 May 2015; accepted 7 July 2015; published 10 July 2015

Copyright (C) 2015 by authors and Scientific Research Publishing Inc.

This work is licensed under the Creative Commons Attribution International License (CC BY).

http://creativecommons.org/licenses/by/4.0/

(c) (i) Open Access

\begin{abstract}
The coexistence of acquired hemophilia $A$ with a secondary retropharyngeal hematoma is an extremely unusual condition with important clinical implications. The purpose of this paper is to present a case involving a patient whose first clinical manifestation, namely dysphagia, along with specific clinical examination and imaging findings, led to an incorrect initial diagnosis of a retropharyngeal abscess. However, performance of a more thorough clinical examination led to the correct diagnosis of a hematoma secondary to acquired hemophilia $A$. This allowed surgery to be avoided in a patient at a high risk of bleeding. Conclusions: Acute neck masses require meticulous differential diagnosis assessing the possible presence of various causative systemic diseases before the most appropriate therapy can be determined.
\end{abstract}

\section{Keywords}

Dysphagia, Cervical Inflammation, Coagulopathy, Hematoma, APTT, Parapharyngeal Spaces

\section{Introduction}

The acute or subacute development of a neck mass usually indicates an infectious or congenital problem. When *Corresponding author.

How to cite this paper: López-Sánchez, I., et al. (2015) Acquired Hemophilia A Simulating Retropharyngeal Abscess: Importance of Differential Diagnosis of Neck Masses before Surgery. International Journal of Otolaryngology and Head \& Neck Surgery, 4, 282-285. http://dx.doi.org/10.4236/ijohns.2015.44048 
acute evolution, poor mass demarcation, and dysphagia develop simultaneously in a patient with a neck mass, the first clinical diagnosis considered is a cervical abscess. One of the less common causes of such a mass is a retropharyngeal hematoma, which is a rare clinical entity [1]. Acquired hemophilia A is an uncommon autoimmune disorder characterized by the presence of autoantibodies to circulating factor VIII [2]. The coexistence of acquired hemophilia A with a secondary retropharyngeal hematoma is an extremely unusual condition with important clinical implications. A literature search of PubMed revealed only one such published case; however, the clinical manifestation involved airway obstruction [3].

The purpose of this paper is to present a case involving a patient whose first clinical manifestation, namely dysphagia, along with specific clinical examination and imaging findings, led to an incorrect initial diagnosis of a retropharyngeal abscess. However, the atypical evolution of the patient's clinical condition and the subsequent performance of a more thorough clinical examination led to the correct diagnosis of a hematoma secondary to acquired hemophilia A. This allowed surgery to be avoided in a patient at a high risk of bleeding.

\section{Case Report}

A 45-year-old woman with no medical history presented to the emergency department with a 48-h history of dysphagia and malaise without other symptoms. Clinical examination revealed thickened lateral and posterior pharyngeal walls extending to the larynx. MRI revealed an extensive inflammatory cervical retropharyngeal abscess extending to the parapharyngeal spaces and upper mediastinum without objectified images of an abscess (Figure 1).

Laboratory examination revealed no signs of infection. The only laboratory abnormalities were a high activated partial thromboplastin time (APTT) (64.2 s; reference range, 24 - $36 \mathrm{~s}$ ) and a low hemoglobin level of 8.3 $\mathrm{g} / \mathrm{dL}$. However, these findings were not considered to be significant at that point in time. Considering these data, the patient was diagnosed with a retropharyngeal abscess with extension to the parapharyngeal spaces. She was hospitalized for intravenous antibiotic treatment and monitoring.

At $48 \mathrm{~h}$ after admission, the patient had developed bruises on her cervical and upper thoracic skin as well as in the posterior pharyngeal wall (Figure 2). Such clinical findings were associated with the previously recognized laboratory abnormality (the prolonged APTT). The Department of Hematology initiated clinical investigation of a possible coagulopathy. The patient had a significantly low level of coagulation factor VIII (4.5\%; reference

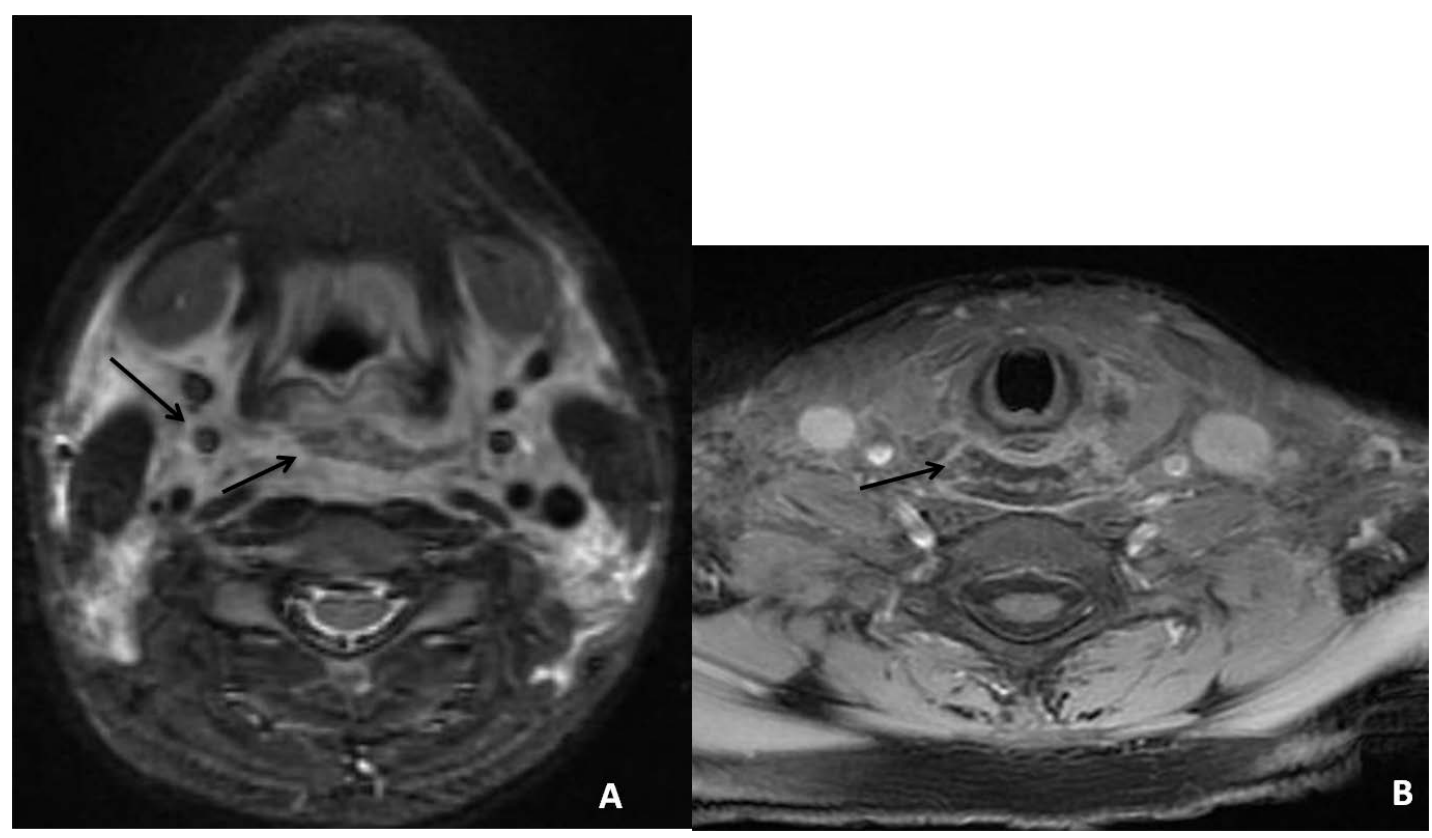

Figure 1. Magnetic resonance imaging: A space-occupying lesion exhibiting diffuse and extensive signal alterations was present in the parapharyngeal, retropharyngeal (A, arrows), and periesophageal spaces (B, arrow). The lesion caused enlargement of the prevertebral space, which extended to all fat cervical planes. These findings were suggestive of an extensive inflammatory cervical abscess extending to the upper mediastinum. 


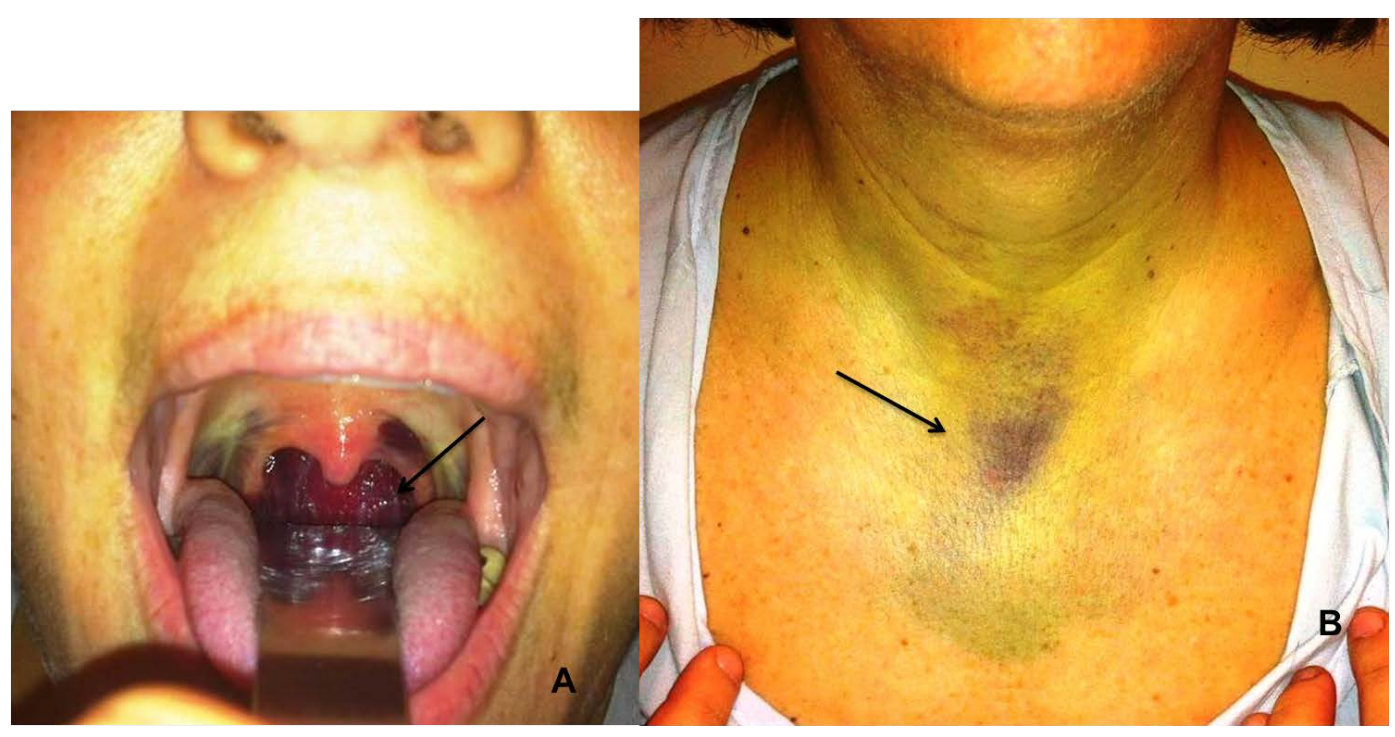

Figure 2. Clinical examination $48 \mathrm{~h}$ after admission. Bruises on the cervical and upper thoracic skin (A, arrow) and in the posterior pharyngeal wall $(B$, arrow) were suggestive of subcutaneous and submucosal hematomas.

range, $70 \%$ - 150\%). Immunologically, the Bethesda test was positive for inhibitors of coagulation factor VIII (1.5 IU Bethesda).

The patient was diagnosed with acquired hemophilia A, and "factor eight inhibitor bypassing activity" (FEIBA) was administered for $24 \mathrm{~h}$. Adjunctive treatment was commenced (corticosteroids and cyclophosphamide). The hemoglobin level increased, and the factor VIII concentration rose to $25 \%$. The patient's clinical symptoms rapidly diminished. A CT scan taken at $48 \mathrm{~h}$ after diagnosis showed a significant reduction in the size of the retropharyngeal hematoma.

After 3 months of monitoring, the APTT and hemoglobin level had normalized, and the factor VIII inhibitory factors had disappeared. She remains clinically asymptomatic at the time of writing.

\section{Discussion}

Diagnosis of acquired hemophilia A is established by the presence of low levels of coagulation factor VIII together with inhibitors of this factor, as determined by the Bethesda test. Our patient met both criteria. In these patients there may be a prolonged activated partial thromboplastin time [4]. Acquired hemophilia A is a serious disease with a mortality rate of up to $9 \%$, mainly because of the development of severe digestive tract and pulmonary hemorrhage (2.5).

Acquired hemophilia A is rare, with an estimated incidence of 1.5 cases per million people per year [5]. The typical manifestation of the disease is development of multiple small foci of spontaneous subcutaneous bleeding [6]. However, manifestation as dysphagia secondary to a retropharyngeal and parapharyngeal hematoma is rare [7].

This case is notable because, initially, our clinical suspicion was a retropharyngeal and parapharyngeal abscess possibly requiring surgical treatment. If an otolaryngologist had decided to perform surgical drainage of the mass based on this initial diagnosis, serious harm to the patient may have resulted from uncontrollable bleeding.

Acute neck masses require meticulous differential diagnosis assessing the possible presence of various causative systemic diseases before the most appropriate therapy can be determined. If the clinical course is not typical and the patient's general condition is not serious, we can delay the surgery a few hours after establishing a medical treatment. During this time we must review all the diagnostic tests carried out to reach a more accurate diagnosis.

\section{Conflict of Interests}

The authors declare no conflict of interest. 


\section{References}

[1] Kubota, H., Endo, H., Noma, M., et al. (2013) Airway Obstruction by a Retropharyngeal Hematoma Secondary to Thoracic Aortic Aneurysm Rupture. Journal of Cardiothoracic Surgery, 8, 232. http://dx.doi.org/10.1186/1749-8090-8-232

[2] Franchini, M., Gandini, G., Di Paolantonio, T. and Mariani, G. (2005) Acquired Hemophilia A: A Concise Review. American Journal of Hematology, 80, 55-63. http://dx.doi.org/10.1002/ajh.20390

[3] Harper, M., Oblensky, L., Roberts, P. and Mercer, M. (2007) A Case of Acute Upper and Lower Airway Obstruction Due to Retropharyngeal Haemorrhage Secondary to Acquired Haemophilia A. Anaesthesia, 6, 627-630. http://dx.doi.org/10.1111/j.1365-2044.2007.05053.x

[4] Freeman, A. (2015) Acquired Haemophilia A Presenting at a District General Hospital. BMJ Case Reports, 17 Feb. 2015, pii: bcr2014208001.

[5] Collins, P.W., Hirsch, S., Baglin, T.P., et al. (2007) Acquired Hemophilia A in the United Kingdom: A 2-Year National Surveillance Study by the United Kingdom Haemophilia Centre Doctors’ Organisation. Blood, 109, 1870-1877. http://dx.doi.org/10.1182/blood-2006-06-029850

[6] Vitry, A., Valois, A., Weinborm, M., et al. (2014) Acquired Haemophilia A: Two Cases. Annales de Dermatologie et de Vénéréologie, 141, 441-445.

[7] Mulliez, S.M., Vantilborgh, A. and Devreese, K.M. (2014) Acquired Hemophilia: A Case Report and Review of the Literature. International Journal of Laboratory Hematology, 36, 398-407. http://dx.doi.org/10.1111/ijlh.12210 